\title{
THE PHILIPPINE RUBIACEAE GENERA: UPDATED SYNOPSIS IN INTKEY DATABASES OF THE DELTA SYSTEM
}

\author{
G.D. ALEJANDRO ${ }^{1} \&$ S. LIEDE 2
}

\begin{abstract}
SUMMARY
Changes in generic concepts and delimitation resulted in 80 genera (6 of which are cultivated) of Philippine Rubiaceae. Generic descriptions of all genera are coded in DELTA format (Version 2000) and translated by the directive CONFOR into INTKEY database for interactive identification and information retrieval. The descriptions incorporate mostly compiled data of Philippine Rubiaceae species and observations on over 130 characters. Recently accepted classification (subfamily, tribes, and subtribes) and significant characters defined by Robbrecht $(1988,1993)$ are appended to the generic descriptions. The database is available on the World Wide Web at http://www.uni-bayreuth.de/ departments/planta2/wgl/delta_ru/index.html.
\end{abstract}

Key words: Rubiaceae, Philippines, DELTA, information retrieval, interactive identification, INTKEY.

\section{INTRODUCTION}

The family Rubiaceae is the fourth largest in number among vascular flora comprising 659 genera and approximately 13,000 species (Robbrecht, 1988). In the Flora Malesiana region, it is estimated only second to the Orchidaceae with about 150 genera and 1,830 species. The most recent enumeration of Philippine Rubiaceae genera is nearly eighty years old (Merrill, 1923) and out of date in many respects. Furthermore, the dearth of generic descriptions essential to the understanding of Rubiaceae in the Philippines prompted us establish an automated databank for Philippine Rubiaceae genera under the DELTA system.

Among dicotyledonous flowering plants, Rubiaceae is the one with the largest number of indigenous species and four endemic genera (Antherostele Bremek., Greeniopsis Merr., Sulitia Merr., and Villaria Rolfe). Merrill (1923) recorded 63 genera and 527 species of Rubiaceae in the 'Enumeration of Philippine Flowering Plants'. However, systematic rearrangements (Bremekamp, 1940b, 1952; Ridsdale 1978c, 1982, 1996; Tirvengadum \& Sastre, 1979; Tirvengadum, 1983; Wong, 1984; Ali \& Robbrecht, 1991; De Block et al., 1996) have resulted in the re-establishment and/or segregation of a number of genera. Recent evaluation for the Philippines resulted in a total of 80 genera (6 of which are cultivated) which comprises $12.1 \%$ of the Rubiaceae genera worldwide. In contrast, the number of species (about 510) has been reduced compared to Merrill's

1) Research Centre for the Natural Sciences, University of Santo Tomas, España Manila, 1008 The Philippines; current address: Department of Plant Systematics, Bayreuth University, 95440 Bayreuth, Germany.

2) Department of Plant Systematics, Bayreuth University, 95440 Bayreuth, Germany. 
(1923) record due to several new synonymies provided in a number of revisions (Cowan, 1936; Bremekamp, 1940a,1947a; Ridsdale 1978a-c, 1996; Jansen \& Ridsdale, 1983; Johansson, 1988; Huxley \& Jebb, 1993; Cabral \& Bacigalupo, 1996).

The present study endeavours to contribute to the understanding of Philippine Rubiaceae. It also ascertains the comparability of this study with other research efforts both on the Philippine flora and on the Taxonomy and Systematics of Rubiaceae worldwide. Moreover, it also validates the new names and combinations with the view to help scientists or foresters interested to get acquainted with the various nomenclatural and taxonomic changes that have occurred in the Rubiaceae of the Philippines. This up-to-date information on the genera of Rubiaceae occurring in the Philippines is an important first step towards a comprehensive treatment of the family for the island and an important contribution to the Flora of the Philippines project, since no recent survey has been done after Merrill's (1923) work.

The lack of comprehensive generic descriptions for Philippine Rubiaceae and the need for an interactive identification tool combined with information retrieval have initiated the use of a DELTA database. The DELTA format is a versatile tool for the storage and management of descriptive taxonomic data (Dallwitz, 1980) while the DELTA system is an integrated set of programs based on the use of the DELTA format. It is advantageous for the first stage in a project as large as the Philippine Rubiaceae, because it is easily accessible and versatile enough to allow corrections and additions throughout the whole project.

\section{MATERIAL AND METHODS}

The DELTA format is mainly composed of 3 files (Dallwitz et al., 1999): 1) a character file (CHARS); 2) a specification file (SPECS), describing character traits like number and type of characters; and 3) a taxon description file (ITEMS). The data are gathered against a character list which can be viewed also on the website. The characters and character states were compiled mostly by consulting literature sources, direct observation, and measurements on herbarium specimens borrowed from the Philippine National Herbarium (PNH), the National Herbarium Netherlands (L), and the National Botanical Garden of Belgium (BR). The list encompasses a total of 139 characters used to generate a natural language description of the Philippine Rubiaceae genera. Likewise, the character list gives information on questions of character definition, and includes other comments which do not appear in the printed taxon descriptions.

For each genus, currently accepted synonyms and type species are given; as well as the subfamiliar, tribal, and subtribal position of the genus according to Robbrecht $(1988,1993)$ and molecular data (Bremer et al., 1995; Bremer, 1996; Bremer \& Thulin, 1998; Bremer \& Manen, 2000; Andreasen \& Bremer, 2000; Razafimandimbison $\&$ Bremer, 2002). Characters include habit and morphology of both vegetative parts and reproductive parts. Further data were added if available and include information on breeding systems, in particular, heterostyly (Bahadur, 1968, 1970), seed anatomy (Robbrecht, 1988, 1993), pollen structure (Robbrecht, 1988, 1993; Puff, 1993) as well as chromosome base number and ploidy level (Kiehn, 1996). Genus distribution, total number of species, and number of Philippine species are indicated as well as important references. 
A sample of the genus descriptions, generated from the databank by CONFOR and typeset automatically, is appended to this article. However, the genus descriptions are continuously improved and revised in the database. Thus, new characters are added from time to time, and existing definitions are improved or rendered less inclusive as more precise data become available. Likewise, characters and character states which are still undesirably inclusive or clumsy will be improved in due course. Against this background, an internet-accessible database has obvious advantages. Moreover, only few images are presently included in the CIMAGES file, and new images are added continuously.

To be able to use the program INTKEY, for interactive identification and information retrieval generated by the directive CONFOR, a PC with Windows 95/NT or later version is needed and the program INTKEY, available at http://biodiversity.uno.edu/delta, must be installed.

\section{RESULTS}

\section{Revisions in Philippine Rubiaceae species}

During the extensive preliminary studies for the Rubiaceae of the Flora of the Philippines project as well as present revisionary work on Philippine Mussaenda Burm. ex L. and Villaria, a number of name and rank changes has become inevitable which will be discussed briefly in the following.

Generic revisions involving Philippine Rubiaceae were initialized by Bremekamp $(1934,1939,1940 a-d, 1947 a, b)$ who published extensive Latin descriptions. Bremekamp $(1934,1939)$ published a monograph of the genus Pavetta L. with emphasis on delimitation against the closely related genus Ixora L. He added four new species of Pavetta to the Philippine record. Six years later, Bremekamp (1940b) published a preliminary account of the Philippine species of Urophyllum Jack ex Wall., Pleiocarpidia Schum. \& Bremek., and Praravinia Korth. \& Bremek. In his investigation of Urophyllum, he noticed four uncommon features : 1) long-pedicellate flowers arising singly or in groups from leaf axils; 2) presence of acaridomatia on the leaves; 3) different kinds of hairs in the corolla; and 4) linear instead of oblong or ovate anther shape. Based on the unusual character of syngenesious anthers, Bremekamp (1940a) introduced a new endemic genus to the Philippines in the same year - Antherostele.

The four genera mentioned are placed in the same tribe, Urophylleae. Moreover, Bremekamp (1940b) revised the genus Williamsia Merr. and included some Urophyllum species in the genus Praravinia. However, he stated that, compared to other genera, delimitation of Urophyllum presents more difficulties. Similarly, he encountered difficulties in the classification of the Philippine species which are imperfectly known to the present day. He refrained from describing several Urophyllum species since the herbarium specimens he studied lack reproductive parts (Bremekamp, 1940b). He also experienced the same difficulty in the treatment of the genus Praravinia and most of the descriptions were based on fruiting material only. In this regard, re-investigation of the closely related Philippine species of Urophyllum and Praravinia is needed.

Moreover, Bremekamp published a monograph on the genus Acranthera Arn. ex Meisn. (1947b) and Streblosa Korth. (1947a). He recognized a single species of Acranthera in the Philippines - A. philippinensis Merr. and added one new endemic 
species and five new varieties of Streblosa. Another early work was made on a systematic arrangement of the closely interrelated Malaysian species of Wendlandia Bartl. ex DC. (Cowan, 1936). Out of four series in Cowan's classification, he grouped the Malaysian Wendlandia in one series - Subinclusae (anthers almost sessile). This series is further subdivided into two subseries, ser. Tinctoriae and ser. Paniculatae, both represented by Philippine species. In 1964, Jayaweera published his treatment of the rubiaceous genus Mussaenda of the Philippine Islands. He added two more Mussaenda species from Merrill's list (1923) endemic to the Philippines and recognized a variety of M. macrophylla Wall. However, Bahadur (1968) remarked that Jayaweera recorded heterostyly in all the Mussaenda species he studied but did not mention dioecism. Heterostyly and dioecism are common on the tribe Mussaendeae (Bahadur, 1968).

Four new genera (Aidia Lour., Rothmannia Thunb., Oxyceros Lour., and Tarennoidea Tirveng. \& Sastre) from the heterogeneous assemblage constituting presently the genus Randia are added to the Philippine Rubiaceae list (Tirvengadum \& Sastre, 1979; Ridsdale, 1996). However, Ridsdale's combinations in Rothmannia and Oxyceros included in Table 1 are not yet published. Fagerlind (1943) observed that revision of Randia s.1. would establish several new genera. Randia is now an exclusively American genus from which eight genera (Aidia, Brachytome, Catunaregam, Ceriscoides, Fagerlindia, Kochummenia, Porterandia, and Rothmannia) were split during a recent revision of the species of the Malay Peninsula (Wong, 1984). Six Philippine species of Randia are still of uncertain position ( $R$. lanceolata, $R$. loheri, $R$. rostrata, $R$. samalensis, $R$. stenophyllum, and R.ticaensis). According to Ridsdale (pers. comm., 2001), Philippine Randia is under study and some of its species will be transferred to the genus Hypobathrum.

The genus Ceriscoides (Hook.f.) Tirveng. has a particularly complex history discussed by Tirvengadum (1978). Tirvengadum (1978, 1983) published studies in the tribe Gardenieae, mostly in the Randia-Gardenia assemblage for tropical Asia. Gardenia Ellis was formerly separated from Randia on the basis of ovary structure (one-celled in Gardenia and two-celled in Randia). Tirvengadum (1978) raised the section Ceriscoides of Randia to generic status accommodating J.D. Hooker's section Ceriscoides of Gardenia. In the Philippines she transferred only one species from Gardenia to Ceriscoides (Tirvengadum, 1983).

Most of the revisions on Philippine Rubiaceae species were made by Ridsdale (1978a-c, 1979, 1982, 1985, 1989, 1996). In 1978 he published a worldwide revision on Mitragyna Korth., Uncaria Schreb., and the tribe Naucleeae (Ridsdale, 1978c) of which 6 genera (Ludekia, Metadina, Myrmeconauclea, Neonauclea, Petrusadina, and Nauclea) are represented in the Philippines. He revised the heterogeneous genus Adina and Philippine representatives were placed under Metadina and Pertusadina. His detailed revisions of Nauclea L. and Neonauclea Merr. (Ridsdale,1978b, c, 1989) have set understanding and delimitations of both genera. He made one combination from Neonauclea to Ludekia, a new genus record to the Philippines. In 1979, he recognized the taxonomic position of Sulitia Merr., an endemic monotypic genus in the Philippines. He transferred the genus from the tribe Cinchoneae to the Gardenieae with reference to the position and form of its inflorescence. Moreover, he published a revision of Badusa (Ridsdale, 1982) describing a new species from Palawan, B. palawensis. However, he recognized $B$. philippica as dubious and taxonomic re-investigation revealed that this species is a synonym of $B$. corymbifera (Soerjarto et al., 1996) found in Tonga, Fiji to Solomon Island, and New Guinea. 
Jansen \& Ridsdale (1983) published a revision of the genus Dolicholobium A. Gray. They recognized the two Philippine representatives as conspecific. In 1985, Ridsdale described a new species of Fagerlindia Tirveng. (F. emanuelssoniana Ridsdale) and a new combination, F. microcarpa (Bartl. ex DC.) Ridsdale (Gardenia micropora). Fagerlindia had been segregated from Randia s.l. by Tirvengadum (1983). Ridsdale's (1996) recent published work deals with a review of Aidia in southeast Asia and Malesia. He described one new species and three new combinations for Philippine record. Ridsdale is currently making combinations in Greeniopsis Merr. and Hymenodictyon Wall. included in Table 1 which will be published in the near future.

Jansen (1984) published a synopsis of Guettardella Champ. ex Benth., where all the Philippine species of Antirhea were transferred to Guettardella as new combinations and one new species (G. caudata) was described. He assigned all Old World Antirhea species to Guettardella except for two species from the Mascarene Islands. Meanwhile, Chaw \& Darwin (1992) made a systematic study of the palaeotropical genus Antirhea including the segregate genus Guettardella. Their extensive study resulted to 36 species of Antirhea, eight out of 13 species in Table 1 are new records for the Philippines.

Other revisions in the 1980s include Knoxia L. (Bhattacharjee \& Deb, 1985; Puff \& Robbrecht, 1989); Prismatomeris Thwaites (Johansson, 1987; Igersheim \& Robbrecht, 1993); and Caelospermum Blume (Johansson, 1988), adding a new Philippine combination ( $C$. volubile) from the closely related genus Morinda L.

Ali \& Robbrecht (1991) published remarks on Asian and Australian taxa included in Diplospora DC. or Tricalysia A. Rich. ex DC. and showed that the two genera form an artificial assemblage of species. The Philippine Tricalysia species were all transferred to Diplospora leaving Tricalysia as an exclusively African genus. Two new combinations in Diplospora were made but the identity of $D$. sessilis is yet to be determined. Discospermum Dalzell which is recognized as a synonym of Diplospora, was upheld at generic rank (Ali \& Robbrecht, 1991). It is represented by only one species, which had been transferred from Gardenia by Ali \& Robbrecht (1991). Huxley \& Jebb (1991a, b, 1993) published a series of tuberous epiphytes recognizing five genera (Anthorrhiza, Hydnophytum, Myrmecodia, Myrmephytum, and Squamellaria) and united them in a new subtribe, the Hydnophytinae, on the base of tuber cavity development and form of inflorescences. Three genera of the tuberous epiphytes (Hydnophytum Jack., Myrmecodia Jack., and Myrmephytum Becc.) are represented in the Philippines. Revisions are available for Myrmephytum (Huxley \& Jebb, 1991b) and Myrmecodia (Huxley \& Jebb, 1993). All previously described Philippine Myrmecodia species were subsumed under one species, M.tuberosa Jack. A revision of Philippine species of Hydnophytum is still wanting. In 1993, the tribal affiliation of Scyphiphora (Puff \& Rohrhofer, 1993) was changed from tribe Knoxieae (subfam. Antirheoideae) to the tribe Gardenieae (subfam. Ixoroideae). Likewise, the tribe Schradereae was re-examined (Puff et al., 1993). Puff et al. (1993) concluded that Lucinaea DC. is a synonym of Schradera Vahl. In a follow-up study (Puff et al., 1998) all previously described Philippine Lucinaea species were transferred to Schradera. One new combination and one new endemic species (Schradera elmeri) were included for the Philippine record. Other generic revisions comprising Philippine genera are those of Xanthophytum Reinw. ex Blume (Axelius, 1990) and Paederia L. (Puff, 1991), but they did not result in name changes or additional records for the Philippines. 
Table 1. New taxa and name changes in Philippine Rubiaceae species since Merrill (1923).

\begin{tabular}{|c|c|c|}
\hline Revised name & Old name & Date of most recent revision \\
\hline Aidia Lour. & & Ridsdale, 1996 \\
\hline A. acuminata (Blume) K.M. Wong & Randia umbellata Elmer & \\
\hline $\begin{array}{l}\text { A. auriculata (Wall.) Ridsdale } \\
\quad \text { var. auriculata }\end{array}$ & Randia auriculata (Wall.) K.Schum. & \\
\hline A. bakeri (Merr.) Ridsdale & Randia bakeri Merr. & \\
\hline $\begin{array}{l}\text { A. foveata Ridsdale } \\
\text { A. pulcherrima (Merr.) Ridsdale }\end{array}$ & Randia pulcherrima Merr. & \\
\hline A. racemosa (Cav.) Tirveng. & Randia racemosa (Cav) Fern.-Vill. & \\
\hline
\end{tabular}

Antirhea Comm. ex Juss.

A. attenuata (Elmer) Chaw

\section{A. benguetensis (Elmer) Valeton \\ A. caudata (M.E. Jansen) Chaw}

A. edanoi Chaw

A. foveolata Chaw

A. hexasperma (Roxb.) Merr.

A. livida Elmer

A. microphylla (Bartl. ex DC.) Merr.

A. paxillata Chaw

A. philippinensis (Benth.) Rolfe

A. ramosii Chaw

A. tayabensis Chaw

A. ternata Chaw

Antherostele Bremek.
A. banahaensis (Elmer) Bremek.
A. callophylla Bremek.
A. grandistipula (Merr.) Bremek.
A. luzoniensis (Merr.) Bremek.

Badusa A. Gray

B. palauensis Valeton

B. palawanensis Ridsdale

B. philippica (Cav.) S.Vidal

Randia racemosa (Cav.) Fern.-Vill.

Antirhea hexasperma Roxb.

Chaw \& Darwin, 1992

Guettardella hexasperma (Roxb.)

M.E. Jansen

Guettardella microphylla (DC.)

M.E. Jansen

Antirhea philippinensis Elmer

Guettardella microphylla (DC.)

M.E. Jansen

Antirhea livida Elmer

Antirhea microphylla (Bartl. ex DC.)

Merr.

Guettardella hexasperma (Roxb.)

M.E. Jansen

Guettardella livida (Elmer) M.E. Jansen

Guettardella microphylla (Bartl. ex DC.)

M.E. Jansen

Antirhea microphylla sensu Merr. Antirhea microphylla sensu Merr.

Antirhea livida sensu Merr.

Urophyllum banahaense Elmer

Bremekamp, 1940b

Urophyllum grandistipulum Merr. Urophyllum luzoniense Merr.

Excluded by Merrill (1923)

Ridsdale, 1982;

Soejarto et al., 1996

B. corymbifera (G.Forst.) A. Gray

Caelospermum Blume (= Coelospermum)

C. volubile (Merr.) J.T. Johanss. Morinda volubilis (Blanco) Merr.

Ceriscoides (Hook.f.) Tirveng.

C. curranii (Merr.) Tirveng. Gardenia curranii Merr.

Chassalia Comm. ex Poir.

C. curviflora (Wall.) Thwaites C.curviflora (Wall.) Thwaites

Johannson, 1988

Tirvengadum, 1983

Deb \& Krishna, 1982

var. ophioxyloides (Wall.) D.B. Deb

\& B. Krishna

Diplospora DC.

D. fasciculiflora (Elmer) Elmer

Randia fasciculiflora Elmer

Ali \& Robbrecht, 1991

D. puberula (Merr.) Ali \& Robbr.

D. sessilis Elmer

Tricalysia puberula Merr.

D. tinagoensis (Elmer) Ali \& Robbr. Tricalysia tinagoensis Elmer 
Table 1 (continued)

\begin{tabular}{llc}
\hline Revised name & Old name & Date of most recent revision \\
\hline $\begin{array}{l}\text { Discospermum Dalzell } \\
\text { D. whitfordii (Elmer) Ali \& Robbr. }\end{array}$ & Gardenia whitfordii Elmer & Ali \& Robbrecht , 1991 \\
$\begin{array}{l}\text { Dolicholobium A. Gray } \\
\text { D. philippinense Trel. }\end{array}$ & Dolicholobium hirsutum Elmer & Jansen \& Ridsdale, 1983
\end{tabular}

Ridsdale, 1985

F. emanuelssoniana Ridsdale

F. microcarpa (Bartl. ex DC.) Ridsdale Gardenia microcarpa Bartl. ex DC.

Greeniopsis Merr.

Ridsdale, unpublished

G. discolor Merr.

G. euphlebia Merr.

G. megalantha Merr.

G. multiflora (Elmer) Merr. Greeniopsis sibuyanensis Elmer

Greeniopsis philippinensis Merr.

Greeniopsis pubescens Merr.

Hymenodictyon Wall.

H. orixense (Roxb.) Mabb.

Ridsdale, unpublished

Knoxia L.

K. sumatrensis (Retz.) DC.

Hymenodictyon excelsum (Roxb.) Wall.

var. sumatrensis

Knoxia corymbosa Willd.

Bhattacharjee \& Deb,

udekia Ridsdale

L. bernardoi (Merr.) Ridsdale

Neonauclea bernardoi Merr.

Ridsdale, $1978 b$

Metadina Bakh.f.

M. trichotoma (Zoll. \& Mor.) Bakh.f. Adina zschokkei Elmer

Ridsdale, 1978b

Mitragyna Korth.

Ridsdale, $1978 \mathrm{c}$

M. diversifolia (Wall. ex G.Don) Havil. Mitragyna rotundifolia (Roxb.) Kuntze

M. speciosa (Korth.) Havil.

Mussaenda Burm. ex L.

M. macrophylla Wall.

var. brevipilosa Jayaw.

M. milleri Elmer

M. pinatubensis Elmer

Myrmecodia Jack

M. tuberosa Jack

M. tuberosa Jack 'apoensis'

C.R. Huxley \& Jebb

M. tuberosa Jack 'sibuyanensis'

C.R. Huxley \& Jebb

Myrmeconauclea Merr.

M. strigosa (Korth.) Merr.

var. fluviatilis Ridsdale

Myrmephytum Becc.

M. beccarii Elmer

M. selebicum (Becc.) Becc.

Nauclea L.

N. orientalis (L.) L.
Mussaenda macrophylla Wall.

Jayaweera, 1964

Huxley \& Jebb, 1993

Myrmecodia echinata Gaudich.

Myrmecodia urdenatensis Elmer

Myrmecodia sorsogonensis Elmer

Myrmecodia apoensis Elmer

Myrmecodia sibuyanensis Elmer

Ridsdale, 1978b

Myrmeconauclea strigosa (Korth.) Merr.

Sarcocephalus fluviatilis Elmer

Huxley \& Jebb, 1991b

Ridsdale, 1978b

Nauclea undulata Roxb.

Nauclea elmerii Merr. 
Table 1 (continued)

\begin{tabular}{lll}
\hline Revised name & Old name & Date of most recent revision
\end{tabular}

Nauclea (continued)

N. robinsonii Merr.

N. subdita (Korth.) Steud. Nauclea junghuhni (Miq.) Merr.

Nauclea multicephala (Elmer) Merr.

Neonauclea Merr.

$N$. bartlingii (DC.) Merr.

var. bartlingii

Old name

Date of most recent revision

var. cumingiana (S.Vidal) Ridsdale

Neonauclea ategii (Elmer) Merr.

Neonauclea vidalii (Elmer) Merr.

Neonauclea cordatula Merr.

N. calycina (DC.) Merr.

Neonauclea monocephala Merr.

$N$. circumscissa Ridsdale

$N$. formicaria (Elmer) Merr.

N. glabra (Roxb.) Bakh.f. \& Ridsdale Neonauclea nitida (Havil.) Merr.

Neonauclea ovata Merr.

$N$.jagorii Merr.

Neonauclea venosa Merr.

N. kentii Merr.

N. lanceolata (Blume) Merr.

subsp. lanceolata

subsp. gracilis (S.Vidal) Ridsdale

Neonauclea mindanaensis Merr.

Neonauclea oligophlebia Merr.

Neonauclea gracilis (S.Vidal) Merr.

N. media (Havil.) Merr.

N.pseudocalycina Ridsdale

$N$. puberula Merr.

Neonauclea forsteri (Seem.) Merr.

N. reticulata (Havil.) Merr.

N. wenzelii Merr.

Oxyceros Lour.

O. bispinosa (Griff.) Tirveng.

Randia uncaria Elmer

Ridsdale, unpublished

Randia williamsii Elmer

Ridsdale, 1978a

Paederia L.

P. foetida L.

P. verticillata Blume

Pavetta L.

P. barnesii Elmer

P. basilanensis Bremek.

P. brachyantha Merr.

P. cumingii Bremek.

P. dolichostyla Merr.

P. elmeri Merr.

$P$. indica $\mathrm{L}$.

P. luzonica Bremek.

P. membranacea Blanco

P. mindanaensis Bremek.

P. parvifolia Vidal

P. phanerophlebia Merr.

P. subferruginea Merr.

P. williamsii Merr.

Puff, 1991

Pertusadina Ridsdale

P. multifolia (Havil.) Ridsdale

Adina multifolia Havil.

Bremekamp, 1934

Pleiocarpidia K. Schum.

P. lanaensis Merr.

Ridsdale, $1978 b$

Bremekamp, 1940a, d 
Table 1 (continued)

\begin{tabular}{lll}
\hline Revised name & Old name & Date of most recent revision
\end{tabular}

Praravinia Korth.

P. acuminata (Merr.) Bremek.

Urophyllum acuminatum Merr.

Bremekamp, 1940a

P. affinis (Merr.) Bremek.

$P$. everettii Merr.

P. glabra (Merr.) Bremek.

Urophyllum affine Merr.

P. loheri (Merr.) Bremek.

Williamsia everettii Merr.

Williamsia glabra Merr.

P. longistipula (Merr.) Bremek.

P. lucbanensis (Elmer) Bremek.

Williamsia loheri Merr.

Williamsia longistipula Merr.

Urophyllum lucbanense Elmer

P. microphylla (Merr.) Bremek.

Urophyllum microphyllum Merr.

P. mimica (Merr.) Bremek.

Williamsia mimica Merr.

P. mindanaensis (Elmer) Bremek. Williamsia mindanaensis Elmer

P. multinervia (Merr.) Bremek. Williamsia multinervia Merr.

P. negrosensis (Merr.) Bremek. Urophyllum negrosense Merr.

P. panayensis (Merr.) Bremek. Williamsia panayensis Merr.

P. pubescens (Quisumb. \& Merr.) Williamsia pubescens Quisumb. \& Merr.

Bremek.

P. quadribracteolata (Merr.) Bremek. Urophyllum quadribrateolatum Merr.

P. sablanensis (Elmer) Bremek. Williamsia sablanensis Elmer

P. stenophylla (Merr.) Bremek. Williamsia stenophylla Merr.

P. triflora (Quisumb. \& Merr.) Bremek. Williamsia triflora Quisumb. \& Merr.

P. viridescens (Elmer) Bremek.

Williamsia viridescens Elmer

Prismatomeris Thwaites

Ridley, 1939;

P. brachypus Ridl.

Johansson, 1987

P. obtusifolia Merr.

P. tetranda (Roxb.) K. Schum. subsp. tetranda J.T. Johanss.

P. tetranda (Roxb.) K. Schum. var. philippinensis Ridl.

Rothmannia Thunb.

$R$. graciliflora (Merr.) Ridsdale

R. lagunensis (Merr.) Ridsdale

$R$. leytensis Ridsdale

R. merrillii (Elmer) Ridsdale

Randia graciliflora Merr.

Ridsdale, unpublished

Gardenia lagunensis Merr.

Gardenia merrillii Elmer

Gardenia negrosensis Merr.

Schradera Vahl (Lucinaea DC.)

S. monocephala (Merr.) Puff,

Buchner \& Greimler

S. elmeri Puff, Buchner \& Greimler

Lucinaea monocephala Merr.

Puff et al., 1998

Lucinaeae epiphytica Elmer

Lucinaea involucrata Elmer

Puff \& Rohrhofer, 1993

Scyphiphora C.F. Gaertn.

Cabral \& Bacigalupo,

= Borreria hispida (L.) K. Schum.

1996

S. hispida L.

S. ocymoides Burm.f.

S. verticillata $\mathrm{L}$.

= Borreria ocymoides (Burm.f.) DC

= Borreria verticillata (L.) G. Mey.

Borreria stricta (L.) G. Mey.

Bremekamp, 1947b

Streblosa glabra Valeton

Streblosa Korth.
S. axilliflora Merr.

var. angustifolia Bremek.

var. latifolia Bremek.

var. laxiflora Bremek.

S. palawanensis Bremek.

var. merrilli Bremek.

var. elmeri Bremek.

Streblosa glabra Valeton 
Table 1 (continued)

\begin{tabular}{|c|c|}
\hline Revised name & Old name \\
\hline $\begin{array}{l}\text { Sulitia Merr. } \\
\text { S. obscurinervia (Merr.) Ridsdale }\end{array}$ & Gardenia obscurinervia Merr \\
\hline $\begin{array}{l}\text { Tarenna Gaertn. } \\
\text { T. bakeri (Merr.) Bremek. } \\
\text { T. luzoniensis (D. Vidal) Bremek. } \\
\text { T. meyeri (Elmer) Bremek. } \\
\text { T. multinervia (Merr.) Bremek. } \\
\text { T. pubescens (Bartl.) Bremek. }\end{array}$ & $\begin{array}{l}\text { Pavetta bakeri Merr. } \\
\text { Pavetta luzoniensis D. Vidal } \\
\text { Pavetta meyeri } \text { Elmer } \\
\text { Pavetta multinervia Merr. } \\
\text { Pavetta pubescens Bartl. }\end{array}$ \\
\hline
\end{tabular}

Tarennoidea Tirveng. \& Sastre

T. wallichii (Hook.f.) Tirveng. \& Sastre Randia wallichii Hook.f.

Tirvengadum \& Sastre, 1979

Uncaria Schreb.

Ridsdale, $1978 \mathrm{c}$

U. acida (Hunter) Roxb. var. acida

U. attenuata Korth.

Uncaria canescens Korth.

U. callophylla Blume ex Korth.

$U$. cordata (Lour.) Merr. forma insignis (DC.) Ridsdale Uncaria insignis DC. forma sundaica Ridsdale

U. lanosa Wall. var. appendiculata (Benth.) Ridsdale forma ferrea (Blume) Ridsdale forma philippinensis (Elmer) Ridsdale forma setiloba (Benth.) Ridsdale Uncaria philippinensis Elmer U. longiflora (Poir.) Merr. var. longiflora var. pteropoda (Miq.) Ridsdale

U. nervosa Elmer

U.perrottetii (A. Rich.) Merr.

$U$. roxburghiana Korth.

$U$. velutina Havil.

Urophyllum Wall.

U. acuminatissimum Merr.

$U$. bataanense Elmer

U. caudatum Merr.

U. elliptifolium Merr.

$U$. leytense Merr.

U.memecycloides (Presl) Rolfe Urophyllum arboreum (Reinw.) Korth.

$U$. mindorense Merr.

$U$. panayense Merr.

U. platyphyllum Elmer

U. reticulatum Elmer

$* U$. spec. aff. reticulatum Identity to be checked

U. subglabrum Merr.

U. urdanetense Elmer

Wendlandia Bartl. ex DC.

Cowan, 1936

W. brachyantha Merr.

W. luzoniensis DC.

Uncaria setiloba Benth.

Uncaria laevifolia Elmer

var. membranifolia (Elmer) Cowan var. williamsii (Merr.) Cowan

Wendlandia membranifolia Elmer

W. nervosa Merr.

W. philippinensis Cowan Wendlandia williamsii Merr.

Wendlandia glabrata DC. 
Table 1 (continued)

\begin{tabular}{l} 
Revised name \\
\hline Wendlandia (continued) \\
W. sibuyanensis Cowan \\
W. syringoides Cowan \\
W. warburgii Merr.
\end{tabular}

Xanthophytum Reinw. ex Blume

Axelius, 1990

$X$. ferrugineum (DC.) Merr.

$X$. fruticulosum Blume

Three other genera (Hedyotis L., Oldenlandia L., and Plectronia L.) included in Merrill's list (1923) are wanting revision. While revising the African Oldenlandia species of tribe Hedyotideae, Bremekamp (1952) split off the genus Exallage Bremek. He refrained from giving a full description of the genus because he could not include a study of the numerous Asian species in his study. In the Philippines, he transferred four species (E.auricularia, E. philippinensis, E. perhispida, and E. radicans) to Exallage out of the 49 Hedyotis species listed by Merrill (1923) and only E. auricularia was provided with a description. The African Exallage is a well-defined genus based on the indehiscent fruit, etc. A similar variation exists in Asian Hedyotis s.1., the identities of which require a meticulous and laborious work to investigate.

In 1985, Bridson reinstated the genus Psydrax after evaluating the heterogeneous African species of Canthium. She divided the genus Psydrax into two subgenera, Psydrax (palaeotropical) and Phallaria (African). She revised only the African species, resulting in 36 species of Psydrax which include new combinations from Canthium and Plectronia. Seven years later, Bridson (1992) published a revision of the genus Canthium in tropical Africa with a complete list of all African epithets of Canthium and Plectronia. Furthermore, she indicated the group affinities of Malesian Canthium which is a good basis for revising the Asian species. The genera Exallage and Psydrax will be included in the Philippine Rubiaceae database when revisions for the Philippine species become available.

Table 1 summarizes all the changes in Philippine Rubiaceae species since Merrill (1923). The old names are written beside currently used names to enable the reader to compare and cross check. Detailed information of all the genera can be retrieved using the INTKEY database.

\section{Taxonomic position of the Philippine Rubiaceae genera}

Classification of the family Rubiaceae has been a point of dispute among contending taxonomic views (Schumann, 1891; Verdcourt, 1958; Bremekamp, 1966; Robbrecht, 1988, 1993) and for molecular data (Bremer et al., 1995; Bremer, 1996; Bremer \& Thulin, 1998; Bremer \& Manen, 2000; Andreasen \& Bremer, 2000; Razafimandimbison $\&$ Bremer, 2002). This large family is still undertreated, many genera needing revision and the complexity of subfamilial and tribal classification remain unsettled (Robbrecht, 1993). 
Table 2. Subfamilial and tribal classification of Philippine Rubiaceae genera.

Subfamilies: ANTIrheoideae, CINChonoideae, COFFeoideae, GUETtardoideae, IXORoideae, POMAzotoideae, RUBIoideae, UROPhylloideae.

Tribes and subtribes: ACRanthereae, ANThospermeae-Coprosminae, ARGostemmateae, CINchoneae, COFfeeae, CONdamineeae, COPtosapeltae, GALieae, GARdenieae-Gardeniinae/ Diplosporinae, GUEttardeae, HAMelieae, HEDyotideae, ISErtieae, IXOreae, KNOxieae, LASiantheae, MORindeae-Morindinae/ Prismatomerideae/Mitchella group, MUSsaendeae, NAUcleeae-Adininae/Mytragyninae/Naucleinae/ Uncarinae, OCTotropideae, OLDenlandieae, OPHiorrhizeae, PAEderieae, PAVetteae, POMazoteae, PSYchotrieae-Psychotriinae/Hydnophytinae, RONdeletieae, RUBieae, SCHradereae, SPErmacoceae, UROphyllea, VANguerieae.

Classification according to different authors: (-), genus not mentioned by author; (?), of uncertain position; $(=)$, same classification as previous author; (*), cultivated; (x), Portlandia group (Robbrecht, 1993).

\begin{tabular}{|c|c|c|c|c|c|}
\hline Genus & $\begin{array}{l}\text { Schumann, } \\
1891\end{array}$ & $\begin{array}{l}\text { Verdcourt, } \\
1958\end{array}$ & $\begin{array}{l}\text { Bremekamp, } \\
1966\end{array}$ & $\begin{array}{l}\text { Robbrecht, } \\
1988,1993\end{array}$ & Molecular data \\
\hline Acranthera & CINC MUS & CINC IXO & IXOR ACR & $\begin{array}{l}\text { CINC ISE, 1988; } \\
\text { Insertae sedis, } 1993\end{array}$ & $(-)$ \\
\hline Aidia & $(-)$ & $(-)$ & $(-)$ & IXOR GAR-G & $(=)$ \\
\hline Amaracarpus & COFF PSY & RUBI PSY & $(=)$ & RUBI PSY-P & RUBI PSY \\
\hline Antherostele & $(-)$ & RUBI URO & UROP URO & CINC URO & $(-)$ \\
\hline Antirhea & COFF GUE & GUET GUE & $(=)$ & ANTI GUE & CINC GUE \\
\hline Argostemma & CINC OLD & RUBI ARG & $(=)$ & $(=)$ & $(=)$ \\
\hline Badusa & CINC CIN & CINC CIN & $(?)$ & $\begin{array}{l}\text { CINC CON, 1988; } \\
\text { (x), } 1993\end{array}$ & $(-)$ \\
\hline Bikkia & CINC CON & CINC RON & CINC CON & $(=), 1988 ;(x), 1993$ & $(-)$ \\
\hline Canthium & $(-)$ & CINC VAN & IXOR VAN & ANTI VAN & $(-)$ \\
\hline Ceriscoides & $(-)$ & $(-)$ & $(-)$ & IXOT GAR-G & $(-)$ \\
\hline Chassalia & COFF PSY & RUBI PSY & $(=)$ & RUBI PSY-P & RUBI PSY \\
\hline Cinchona* & CINC CIN & $(=)$ & $(=)$ & $(=)$ & $(-)$ \\
\hline Coelospermum & COFF MOR & RUBI MOR & RUBI MOR & $(=)$ & RUBI MOR-Mo \\
\hline Coffea* & COFF IXO & CINC IXO & IXOR COF & IXOR COF & $(=)$ \\
\hline Coptosapelta & CINC CIN & CINC CIN & IXOR COP & CINC COP & $(-)$ \\
\hline Cowiea & $(-)$ & $(-)$ & $(-)$ & IXOR OCT & $(-)$ \\
\hline Damnacanthus & CINC MOR & RUBI MOR & $(=)$ & RUBI MOR-Mi & $(=)$ \\
\hline Dentella & CINC OLD & RUBI HED & $(=)$ & $(=)$ & RUBI SPE \\
\hline Diplospora & CINC GAR & $(=)$ & IXOR GAR & IXOR GAR-D & IXOR COF \\
\hline Discospermum & $(-)$ & $(-)$ & $(-)$ & IXOR GAR-D & IXOR COF \\
\hline Dolicholobium & CINC CIN & $(=)$ & $(=)$ & $(=)$ & $(-)$ \\
\hline Exallage & $(-)$ & $(-)$ & RUBI HED & $(=)$ & $(-)$ \\
\hline Fagerlindia & $(-)$ & $(-)$ & $(-)$ & IXOR GAR-G & $(-)$ \\
\hline Galium & COFF GAL & RUBI RUB & $(=)$ & $(=)$ & $(=)$ \\
\hline Gardenia & CINC GAR & $(=)$ & IXOR GAR & IXOR GAR-G & $(=)$ \\
\hline Geophila & COFF PSY & RUBI PSY & $(=)$ & RUBI PSY-P & RUBI PSY \\
\hline Greenea & CINC RON & $(=)$ & $(=)$ & $(=)$ & $(-)$ \\
\hline Greeniopsis & $(-)$ & $(-)$ & $(-)$ & CINC COP & $(-)$ \\
\hline Guettarda & COFF GUE & GUET GUE & $(=)$ & ANTI GUE & CINC GUE \\
\hline Gynochthodes & COFF MOR & RUBI MOR & $(=)$ & $(=)$ & RUBI MOR-Mo \\
\hline Hamelia* & CINC GAR & RUBI HAM & $(=)$ & $(=)$ & CINC HAM \\
\hline Hedyotis & $(-)$ & RUBI HED & $(=)$ & $(=)$ & RUBI SPE \\
\hline Hydnophytum & COFF PSY & RUBI PSY & $(=)$ & RUBI PSY-H & RUBI PSY \\
\hline Hymenodictyon & CINC CIN & $(=)$ & $(=)$ & CINC COP & $(-)$ \\
\hline Hypobathrum & CINC GAR & $(=)$ & IXOR GAR & IXOR OCT & $(-)$ \\
\hline Ixora & COFF IXO & CINC IXO & IXOR COF & IXOR PAV & IXOR IXO \\
\hline Knoxia & COFF KNO & RUBI KNO & $(=)$ & ANTI KNO & RUBI SPE \\
\hline
\end{tabular}


Table 2 (continued)

\begin{tabular}{|c|c|c|c|c|c|}
\hline Genus & $\begin{array}{l}\text { Schumann, } \\
1891\end{array}$ & $\begin{array}{l}\text { Verdcourt, } \\
1958\end{array}$ & $\begin{array}{l}\text { Bremekamp, } \\
1966\end{array}$ & $\begin{array}{l}\text { Robbrecht, } \\
1988,1993\end{array}$ & Molecular data \\
\hline Lasianthus & COFF PSY & RUBI PSY & $(=)$ & $\begin{array}{l}\text { RUBI PSY-P (ten- } \\
\text { tatively included) }\end{array}$ & RUBI LAS \\
\hline Ludekia & $(-)$ & $(-)$ & $(-)$ & CINC NAU-A & $(=)$ \\
\hline Metadina & $(-)$ & $(-)$ & $(-)$ & CINC NAU-A & $(=)$ \\
\hline Mitragyna & CINC NAU & CINC CIN & $(=)$ & CINC COP & CINC NAU-M \\
\hline Morinda & COFF MOR & RUBI MOR & $(=)$ & $(=)$ & RUBI MOR-Mo \\
\hline Mussaenda & CINC MUS & $(=)$ & $(=)$ & CINC ISE & IXOR MUS \\
\hline Mycetia & CINC MUS & RUBI HED & $(=)$ & CINC ISE & RUBI ARG \\
\hline Myrmecodia & COFF PSY & RUBI PSY & $(=)$ & RUBI PSY-H & RUBI PSY \\
\hline Myrmeconauclea & $(-)$ & $(-)$ & $(-)$ & CINC NAU-A & $(=)$ \\
\hline Myrmephytum & COFF PSY & RUBI PSY & $(=)$ & RUBI PSY-H & RUBI PSY \\
\hline Nauclea & CINC NAU & $(=)$ & $(=)$ & CINC NAU-N & $(=)$ \\
\hline Neonauclea & $(-)$ & CINC CIN & $(=)$ & CINC NAU-A & $(=)$ \\
\hline Nertera & COFF ANT & RUBI ANT & $(=)$ & RUBI ANT-C & RUBI ANT \\
\hline Oldenlandia & CINC OLD & RUBI HED & RUBI HED & $(=)$ & RUBI SPE \\
\hline Ophiorrhiza & CINC OLD & RUBI OPH & UROP OPH & RUBI OPH & $(=)$ \\
\hline Oxyceros & $(-)$ & $(-)$ & $(-)$ & IXOR GAR-G & $(=)$ \\
\hline Paederia & COFF PAE & RUBI PAE & RUBI PAE & $(=)$ & $(=)$ \\
\hline Pavetta & COFF IXO & CINC IXO & IXOR IXO & IXOR PAV & $(=)$ \\
\hline Pentas* & CINC OLD & RUBI HED & $(=)$ & $(=)$ & RUBI SPE \\
\hline Pertusadina & $(-)$ & $(-)$ & $(-)$ & CINC NAU-A & $(=)$ \\
\hline Pleiocarpidia & $(-)$ & RUBI URO & UROP URO & CINC URO & $(-)$ \\
\hline Posoqueria* & CINC GAR & $(=)$ & $(?)$ & IXOR GAR-G & (?) \\
\hline Praravinia & CINC MUS & RUBI URO & UROP URO & CINC URO & RUBI URO \\
\hline Prismatomeris & COFF MOR & RUBI MOR & $(=)$ & RUBI MOR-P & $(=)$ \\
\hline Psychotria & COFF PSY & RUBI PSY & $(=)$ & RUBI PSY-P & RUBI PSY \\
\hline Psydrax & $(-)$ & $(-)$ & $(-)$ & ANTI VAN & IXOR VAN \\
\hline Rondeletia* & CINC RON & $(=)$ & $(=)$ & $(=)$ & $(-)$ \\
\hline Rothmannia & $(-)$ & $(-)$ & $(-)$ & IXOR GAR-G & $(=)$ \\
\hline Rubia & COFF GAL & RUBI RUB & $(=)$ & $(=)$ & $(=)$ \\
\hline Saprosma & COFF PSY & RUBI PAE & $(=)$ & $(=)$ & $(-)$ \\
\hline Schradera & CINC MUS & RUBI SCH & $(=)$ & $(=)$ & $(=)$ \\
\hline Scyphiphora & CINC GAR & RUBI KNO & RUBI & ANTI KNO & $(-)$ \\
\hline Spermacoce & COFF SPE & RUBI SPE & $(=)$ & $(=)$ & $(=)$ \\
\hline Streblosa & $(-)$ & RUBI PSY & $(=)$ & RUBI PSY-P & $(-)$ \\
\hline Sulitia & $(-)$ & $(-)$ & $(-)$ & IXOR GAR-G & $(-)$ \\
\hline Tarenna & $(-)$ & CINC IXO & IXOR IXO & IXOR PAV & $(=)$ \\
\hline Tarennoidea & $(-)$ & $(-)$ & $(-)$ & IXOR GAR-G & $(-)$ \\
\hline Timonius & COFF GUE & GUET GUE & $(=)$ & ANTI GUE & $(-)$ \\
\hline Uncaria & $(-)$ & CINC CIN & $(=)$ & CINC COP & CINC NAU-U \\
\hline Urophyllum & CINC MUS & RUBI URO & UROP URO & CINC URO & RUBI URO \\
\hline Villaria & CINC GAR & $(=)$ & IXOR GAR & IXOR OCT & $(-)$ \\
\hline Wendlandia & CINC RON & $(=)$ & $(=)$ & $(=)$ & $(-)$ \\
\hline Xanthophytum & CINC OLD & RUBI OPH & POMA POM & RUBI HED & RUBI OPH \\
\hline
\end{tabular}


The latest accepted classification of Robbrecht $(1988,1993)$ is basically adopted here but molecular phylogenetic results were also incorporated. Refer to Table 2 for quick identification of Philippine Rubiaceae genera placements which are also included in the generic descriptions of the INTKEY database. The four subfamilies (Cinchonoideae, Ixoroideae, Antirheoideae, and Rubioideae) recognized by Robbrecht are all represented in Philippine Rubiaceae genera, subfam. Rubioideae being the largest in genus numbers. Of 44 tribes (five of which are tribus incertae as to subfamiliar placement) in his list (Robbrecht, 1988), a total of 25 tribes are found in the Philippines.

In contrast to Robbrecht's $(1988,1993)$ classification, molecular data of Bremer (1996) suggest only three subfamilies (Cinchonoideae, Ixoroideae, and Rubioideae). Phylogenetic results showed that the members of subfam. Antirheoideae are nested among the three other subfamilies. Likewise, she made some modifications of the tribal groupings particularly in the subfamily Rubioideae. However, Bremer (1996) stated that there is a need for more genera of Rubiaceae to be analyzed to obtain strong evidence and relevant data for subfamilial revisions of Rubiaceae classification. Indeed, the impact of phylogenetic analyses show that Rubiaceae is still systematically unsettled.

\section{ACKNOWLEDGEMENTS}

We gratefully acknowledge Deutscher Akademischer Austauschdienst (DAAD) for the scholarship award without which the project would not have been possible. Dr. Colin E. Ridsdale for generously sharing his knowledge of Rubiaceae. PNH, L, and BR herbaria for lending specimens. Blumea editors for allowing the use of Rubiaceae illustrations from the Blumea journal on the website. Dr. Petra de Block for providing various literature. Dr. Ulrich Meve for sharing his thoughts on the field of taxonomy. Dr. Andreas Jürgens for his unending generous assistance in everything especially in putting up the website. Irma Mika for her help with the Latin diagnoses and to all the staff of the Department of Plant Systematics, University of Bayreuth.

\section{REFERENCES}

Ali, S.I. \& E. Robbrecht. 1991. Remarks on the tropical Asian and Australian taxa included in Diplospora or Tricalysia (Rubiaceae-Ixoroideae-Gardenieae). Blumea 35: 279-305.

Andreasen K. \& B. Bremer. 2000. Combined phylogenetic analysis in the Rubiaceae-Ixoroideae: morphology, nuclear and chloroplast DNA data. Amer. J. Bot. 87: 1731-1748.

Axelius, B. 1990. The genus Xanthophytum (Rubiaceae). Taxonomy, phylogeny and biogeography. Blumea 34: 425-497.

Bahadur, B. 1968. Heterostyly in Rubiaceae: A review. J. Osmania Univ. (Sci.): 207-238.

Bahadur, B. 1970. Heterostyly and homostyly in Pentas lanceolata (Forsk.) Delf. J. Genetics 60: 199-204.

Bhattacharjee, R. \& D.B. Deb. 1985. A revision of Knoxia (Rubiaceae). J. Econ. Tax. Bot. 6: $73-95$.

Bremekamp, C.E.B. 1934. A monograph of the genus Pavetta L. Feddes Repert. Spec. Nov. Regni Veg. 37: 1-208.

Bremekamp, C.E.B. 1939. A monograph of the genus Pavetta L.: additions and emendations. Feddes Repert. Spec. Nov. Regni Veg. 47: 12-28, 81-98.

Bremekamp, C.E.B. 1940a. Antherostele genus novum Rubiacearum Urophyllo affine. J. Arnold Arbor. 21: 25-31.

Bremekamp, C.E.B. 1940b. A preliminary account of the Philippine species of Urophyllum Wall., Pleiocarpidia K. Schum. \& Praravinia Korth. (Rubiaceae). J. Arnold Arbor. 21: 32-47. 
Bremekamp, C.E.B. 1940c. On Urophyllum Wall. (Rubiaceae) and its nearest allies. Recueil Trav. Bot. Neerl. 37: 171-197.

Bremekamp, C.E.B. 1940d. A monograph of the genus Pleiocarpidia K. Sch. (Rubiaceae). Recueil Trav. Bot. Neerl. 37: 198-236.

Bremekamp, C.E.B. 1947a. A monograph of the genus Streblosa Korth. (Rubiaceae). J. Arnold Arbor. 28: 145-185.

Bremekamp, C.E.B. 1947b. A monograph of the genus Acranthera Arn. ex Meisn. J. Arnold Arbor. 28: 261-308.

Bremekamp, C.E.B. 1952. The African species of Oldenlandia L. sensu Hiern et K. Schumann. Verh. Kon. Ned. Akad. Wet., Afd. Natk., 2e reeks, 48: 1-297.

Bremekamp, C.E.B. 1966. Remarks on the position, the delimitation and the subdivision of the Rubiaceae. Acta Bot. Neerlandica 15: 1-33.

Bremer, B. 1996. Phylogenetic studies within Rubiaceae and relationships to other families based on molecular data. Opera Bot. Belg. 7: 33-50.

Bremer, B., K. Andreasen \& D. Olsson. 1995. Subfamilial and tribal relationships in the Rubiaceae based on rbcL sequence data. Ann. Missouri Bot. Gard. 82: 383-397.

Bremer, B. \& J.F. Manen. 2000. Phylogeny and classification of the subfamily Rubioideae (Rubiaceae). Pl. Syst. Evol. 225: 43-72.

Bremer, B. \& M. Thulin. 1998. Collapse of Isertieae, re-establishment of Mussaendeae, and a new genus of Sabiceeae (Rubiaceae); phylogenetic relationships based on rbcL data. Pl. Syst. Evol. 211: 71-92.

Bridson, D.M. 1985. The reinstatement of Psydrax (Rubiaceae, subfam. Cinchonoideae tribe Vanguerieae) and a revision of the African species. Kew Bull. 40: 687-725.

Bridson, D.M. 1992. The genus Canthium (Rubiaceae-Vanguerieae) in tropical Africa. Kew Bull. 47: 353-401.

Cabral,E.L. \& N.M. Bacigalupo. 1996. Revision of Borreria section Pseudodiodia (Rubiaceae-Spermacoceae). Opera Bot. Belg. 7: 309-327.

Chaw, S.M. \& S.P. Darwin. 1992. A systematic study of the palaeotropical genus Antirhea (Rubiaceae: Guettardeae). Tulane Stud. Zool. Bot. 28: 25-118.

Cowan, J.M. 1936. The Malaysian species of Wendlandia (Rubiaceae). Bull. Jard. Bot. Buitenzorg III, 14: 8-46.

Dallwitz, M.J. 1980. A general system for coding taxonomic descriptions. Taxon 29: 41-46.

Dallwitz, M.J., T.A. Paine \& E.J. Zurcher. 1999 onwards. User's guide to the DELTA Editor. http://biodiversity.uno.edu/delta/

De Block, P., E. Robbrecht \& C. Puff. 1996. Advances in Rubiaceae research. Opera Bot. Belg. 7: $19-32$.

Deb, D.B. \& B. Krishna. 1982. Taxonomic studies of the genus Chassalia Comm. ex Poir. in India and adjoining regions. Bull. Bot. Surv. India 24: 221-224.

Fagerlind, F. 1943. Die Sprossfolge in der Gattung Randia und ihre Bedeutung für die Revision der Gattung. Ark. Bot. 30 A: 1-57.

Huxley, C.R. \& M.H. Jebb. 1991a. The tuberous epiphytes of the Rubiaceae 1: A new subtribe - The Hydnophytinae. Blumea 36: 1-20.

Huxley, C.R. \& M.H. Jebb. 1991b. The tuberous epiphytes of the Rubiaceae 3: A revision of Myrmephytum to include Myrmedoma. Blumea 36: 43-52.

Huxley, C.R \& M.H. Jebb. 1993. The tuberous epiphytes of the Rubiaceae 5: A revision of Myrmecodia. Blumea 37: 271-334.

Igersheim, A. \& E. Robbrecht. 1993. The character states and relationships of the Prismatomerideae (Rubiaceae-Rubioideae). Opera Bot. Belg. 6: 61-79.

Jansen, M.E. 1984. A synopsis of Guettardella Benth. and the Old World species of Antirhea (Rubiaceae: Guettardeae). Blumea 29: 565-588.

Jansen, M.E. \& C.E. Ridsdale. 1983. A revision of the genus Dolicholobium (Rubiaceae). Blumea 29: $251-311$.

Jayaweera, D.M.A. 1964. The Rubiaceous genus Mussaenda: The species of the Philippine Islands. J. Arnold Arbor. 45: 101-139. 
Johansson, J.T. 1987. The revision of the genus Prismatomeris Thw. (Rubiaceae, Morindeae). Opera Botanica 94: 5-62.

Johansson, J.T. 1988. Revision of the genus Caelospermum (Rubiaceae, Rubioideae, Morindeae). Blumea 33: 265-297.

Kiehn, M. 1996. Chromosomes of Rubiaceae occurring in Malesia, the Philippines, New Guinea, and the Pacific. Opera Bot. Belg. 7: 249-260.

Merrill, E.D. 1923. An enumeration of Philippine flowering plants. Manila Bureau of Printing 2: 492-576.

Puff, C. 1991. Revision of the genus Paederia L. (Rubiaceae-Paederieae) in Africa and Madagascar. Opera Bot. Belg. 3: 293-322.

Puff, C. 1993. Pollen nuclear numbers in the Rubiaceae. Opera Bot. Belg. 6: 31-49.

Puff, C., L. Andersson, U. Rohrhofer \& A. Igersheim. 1993. The tribe Schradereae (Rubiaceae) reexamined. Bot. Jahrb. Syst. 114: 449-479.

Puff, C., J. Greimler \& R. Buchner. 1998. Revision of Schradera (Rubiaceae-Schradereae) in Malesia. Blumea 43: 287-335.

Puff, C. \& E. Robbrecht. 1989. A survey of the Knoxieae (Rubiaceae-Antirheoideae). Bot. Jahrb. Syst. 110: 511-588.

Puff, C., E. Robbrecht \& L. Andersson. 1991. Relationships and evolution in Paederia L. (Rubiaceae-Paederieae). A comparison classical approach, numerical evaluation and cladistic analysis . Opera Bot. Belg. 3: 337-358.

Puff, C. \& U. Rohrhofer. 1993. The character states and taxonomic position of the monotypic mangrove genus Scyphiphora (Rubiaceae). Opera Bot. Belg. 6: 143-172.

Razafimandimbison, S., \& B. Bremer. 2002. Phylogeny and classification of Naucleeae s.1. (Rubiaceae) inferred from molecular (ITS, rbcL and trnT-F) and morphological data. Amer. J. Bot. 89: 1027-1041.

Ridley, H.N. 1939. Morindeae in notes on some Malayan Rubiaceae. Kew Bull: 600-611.

Ridsdale, C.E. 1978a. A revision of Mitragyna \& Uncaria. Blumea 24: 43-100.

Ridsdale, C.E. 1978b. A revision of Neonauclea (Rubiaceae). Blumea 24: 177-275.

Ridsdale, C.E. 1978c. A revision of the tribe Naucleeae s.s. (Rubiaceae). Blumea 24: 307-366.

Ridsdale, C.E. 1979. The taxonomic position of Sulitia (Rubiaceae). Blumea 25: 301-303.

Ridsdale, C.E. 1982. A revision of the genus Badusa (Rubiaceae, Condamineeae, Porlandiinae). Blumea 28: 145-150.

Ridsdale, C.E. 1985. The genus Fagerlindia (Rubiaceae) in the Philippines. Blumea 31: 239-244.

Ridsdale, C.E. 1989. A revision of Neonauclea (Rubiaceae). Blumea 34: 177-275.

Ridsdale, C.E. 1996. A review of Aidia s.1. (Rubiaceae) in Southeast Asia and Malesia. Blumea 41: $135-179$.

Robbrecht, E. 1988. Tropical woody Rubiaceae. Opera Bot. Belg. 1:1-271.

Robbrecht, E. 1993. Supplement to the 1988 outline of the classification of the Rubiaceae index to genera. Opera Bot. Belg. 6: 173-196.

Schumann, K. 1891. Rubiaceae. In: A. Engler \& K. Prantl (eds.), Die natürlichen Pflanzenfamilien, vol. 4, 4: 1-156.

Soejarto, D., P. Delprete, J.C. Regalado \& D.A. Madulid. 1996. The true provenance and identity of Badusa philippica (Rubiaceae). Taxon 45: 487-492.

Tirvengadum, D.D. 1978. A synopsis of the Rubiaceae-Gardenieae of Ceylon (Sri Lanka). Adansonia 35: 3-33.

Tirvengadum, D.D. 1983. New taxa and name changes in tropical Asiatic Rubiaceae. Nordic J. Bot. 3: $455-469$.

Tirvengadum, D.D. \& C. Sastre. 1979. La signification taxonomique des modes des ramification de Randia et genres affines. Mauritius Inst. Bull. 8: 77-98.

Verdcourt, B. 1958. Remarks on the classification of the Rubiaceae. Bull. Jard. Bot. État. 28: $209-281$.

Wong, K.M. 1984. The genera of Peninsular Malaysian Rubiaceae formerly confused with Randia. Malayan Nat. J. 38: 1-57. 


\section{APPENDIX: EXAMPLE FOR A GENERIC DESCRIPTION PRODUCED BY DELTA}

\section{Mussaenda Burm. ex L.}

Type species: Mussaenda frondosa $\mathrm{L}$.

Classification: Subfamily: Ixoroideae; Tribe: Mussaendeae (update from Bremer \& Thulin, 1998).

Habit and vegetative parts: Trees, shrubs or undershrubs (rarely herbaceous), 1-8 $\mathrm{m}$ high, without succulent tuber. Spines absent. Raphides absent. Vegetative parts not foetid when bruised. Stem glabrous or pubescent, branched. Branches and branchlets pubescent (rarely glabrous). Adventitious roots on internodes absent. Stipules interpetiolar, not fused, entire to bifid, small to large, ovate to lanceolate or elliptic, pubescent, margin not hairy, colleters present (at the base), persistent or caducous. Leaves opposite or whorled (ternately), isophyllous or anisophyllous. Lamina membranous to papery, 4-20 $(-30) \mathrm{cm}$ long, $2-10(-16.5) \mathrm{cm}$ wide, ovate-lanceolate, or obovate; apex acute or acuminate or obtuse; base cuneate or obtuse, or rounded, decurrent on petiole or not; not ciliated on the margins; upper surface glabrous or pubescent, sparse; lower surface glabrous or pubescent, sparse or dense; subsessile or petiolate; lateral nerves prominent, 5-13(-18) pairs. Petiole 0.5-4(-7.5) cm long, pubescent, sparse or dense, not winged. Domatia absent.

Reproductive parts: Inflorescences present, axillary or terminal; flowering heads fascicled, on a branched axis; inflorescences cymes or thyrses; involucral bracts absent; interfloral bracts and bracteoles present. Flowers 5-merous, actinomorphic, bisexual, heterostylous, with semaphylls, not fragrant; pedicel absent or present, pubescent. Calyx tube extremely reduced $(<2 \mathrm{~mm}$ long) or cup-shaped to shortly tubular ( $>2 \mathrm{~mm}$ long), hairs present; lobes short to well-developed ( $>2 \mathrm{~mm}$ ), equal, pubescent; colleters present. Corolla salverform or infundibular, $2-10 \mathrm{~cm}$ long; tube pubescent outside and inside; lobes linear-lanceolate or ovate; aestivation valvate; abaxial side pubescent; adaxial side pubescent; margin hairy or not hairy. Stamens inserted distal $1 / 4$ of corolla tube or around middle (1/4-3/4 of corolla tube), separate, of equal sizes. Filaments distinct ( $>1 \mathrm{~mm}$ ), not hairy. Anthers dorsifixed, included in corolla tube, linear, dehiscing via longitudinally slits. Style included in corolla tube, terete or filiform, hairs present or absent. Stigmas lobate, 2, fusiform or linear, papillate. Ovary inferior, bilocular; placentation axile. Ovule per locule numerous, not pendulous. Fruits fleshy, not multiple, non-pyreniferous, berry, globose or ellipsoid; ridges or grooves absent; crown of calyx segments present or absent. Seeds numerous, angular, wingless, endospermic; exotestal cells isodiametric, with thickenings along the radial wall or in the inner tangential walls; isolated fibers absent.

Pollen binucleate, aperture 3 to $>3$; colporate or porate.

Chromosome basic number $\mathrm{x}=11$, ploidy level 2 .

Distribution - extending through the Old World tropics from Africa \& Madagascar to India \& Ceylon, China, Malaysia, the Philippines, Polynesia, New Guinea, and Australia.

Number of species -190 .

Philippine species - *M. acuminatissima Merr., ${ }^{*}$ M. albiflora Merr., *M. anisophylla Vidal, *M. attenuifolia Elmer, ${ }^{*} M$. benguetensis Elmer, ${ }^{*} M$. chlorantha Merr., ${ }^{*} M$. grandifolia Elmer, ${ }^{*}$ M. lanata C.B. Rob., M. macrophylla Wall. var. brevipilosa Jayaweera, *M. magallanensis Elmer, M. milleri Elmer, ${ }^{*}$ M. multibracteata Merr., *M. nervosa Elmer, *M. palawanensis Merr., *M. philippica A. Rich., ${ }^{*}$ M. philippica forma aurorae (Sulit), ${ }^{*}$ M. philippinensis Merr., *M. pinatubensis Elmer, ${ }^{*}$ M. scandens Elmer, *M. setosa Merr., *M. vidalii Elmer (*endemic).

Useful literature - Jayaweera, D.M.A. 1963. The Rubiaceous genus Mussaenda: The morphology of the Asiatic species. J. Arn. Arb. 44: 111-126. Jayaweera, D.M.A. 1963. The Rubiaceous genus Mussaenda: The species of the Philippine Islands. J. Arn. Arb. 45: 101-139. Puff, C. et al. 1993. Pseudomussaenda and Schizomussaenda (Rubiaceae): close allies of Mussaenda. Bul. Jard. Bot. Nat. Belg. 62: 35-68. Andersson, L. 1996. Circumscription of the tribe Isertieae (Rubiaceae). Opera Bot. Belg. 7: 139-164. Bremer, B. \& M. Thulin. 1998. Collapse of Isertieae, re-establishment of Mussaendeae, and a new genus of Sabiceeae (Rubiaceae); phylogenetic relationships based on rbcL data. Pl. Syst. Evol. 211: 71-92. 